(C) 2020. The American Astronomical Society. All rights reserved. Access to this work was provided by the University of Maryland, Baltimore County (UMBC) ScholarWorks@UMBC digital repository on the Maryland Shared Open Access (MD-SOAR) platform.

Please provide feedback

Please support the ScholarWorks@UMBC repository by emailing scholarworks-group@umbc.edu and telling us what having access to this work means to you and why it's important to you. Thank you. 
Draft version September 18, 2020

Preprint typeset using $\mathrm{LAT}_{\mathrm{E}} \mathrm{X}$ style emulateapj v. 12/16/11

\title{
SEARCH FOR HIGH-REDSHIFT BLAZARS WITH FERMI/LAT
}

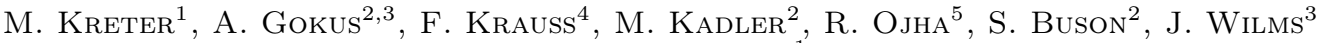 \\ AND M. BÖTTCHER ${ }^{1}$ \\ ${ }^{1}$ Centre for Space Research, North-West University, Private Bag X6001, Potchefstroom 2520, South Africa \\ ${ }^{2}$ Lehrstuhl für Astronomie, Universität Würzburg, Emil-Fischer-Strasse 31, 97074 Würzburg, Germany \\ ${ }^{3}$ Dr. Remeis Sternwarte \& ECAP, Universität Erlangen-Nürnberg, Sternwartstrasse 7, 96049 Bamberg, Germany \\ ${ }^{4}$ Department of Astronomy \& Astrophysics, Pennsylvania State University, University Park, PA, 16802, United States and \\ ${ }^{5}$ NASA Goddard Space Flight Center, Greenbelt, MD 20771, USA \\ Draft version September 18, 2020
}

\begin{abstract}
High- $z$ blazars $(z \geq 2.5)$ are the most powerful class of persistent $\gamma$-ray sources in the Universe. These objects possess the highest jet powers and luminosities and have black hole masses often in excess of $10^{9}$ solar masses. In addition, high- $z$ blazars are important cosmological probes and serve as test objects for blazar evolution models. Due to their large distance, their high-energy emission typically peaks below the GeV range, which makes them difficult to study with Fermi/LAT. Therefore, only the very brightest objects are detectable and, to date, only a small number of high-z blazars have been detected with Fermi/LAT.

In this work, we studied the monthly binned long-term $\gamma$-ray emission of a sample of 176 radio and optically detected blazars that have not been reported as known $\gamma$-ray sources in the 3FGL catalog. In order to account for false-positive detections, we calculated monthly Fermi/LAT light curves for a large sample of blank sky positions and derived the number of random fluctuations that we expect at various test statistic (TS) levels. For a given blazar, a detection of TS $>9$ in at least one month is expected $\sim 15 \%$ of the time. Although this rate is too high to secure detection of an individual source, half of our sample shows such single-month $\gamma$-ray activity, indicating a population of highenergy blazars at distances of up to $\mathrm{z}=5.2$. Multiple $\mathrm{TS}>9$ monthly detections are unlikely to happen by chance, and we have detected several individual new sources in this way, including the most distant $\gamma$-ray blazar, BZQ J1430 $+4204(\mathrm{z}=4.72)$. Finally, two new $\gamma$-ray blazars at redshifts of $\mathrm{z}=3.63$ and $\mathrm{z}=3.11$ are unambiguously detected via very significant (TS $>25$ ) flares in individual monthly time bins.
\end{abstract}

\section{INTRODUCTION}

The most luminous sources across the entire electromagnetic spectrum are active galactic nuclei (AGN). A fraction of AGN exhibit a collimated outflow of material from the central region, a jet. Depending on the orientation of an AGN on the night sky, we see its jets from different angles. An AGN with a jet pointing towards Earth is called a blazar and, due to the relativistic speed of the material in the jet, its emission appears brighter and more variable compared to other AGN. Due to the extreme luminosities of blazars, it is possible to observe them even at large redshifts. Studies by Ajello et al. (2009); Toda et al. (2020) revealed that the density of the most powerful blazars appears to peak at $\mathrm{z} \sim 4$. These high- $z$ blazars seem to harbor supermassive black holes with masses often in excess of $10^{9} M_{\odot}$ (Bloemen et al. 1995; Ghisellini et al. 2010), which means they have larger masses than most blazars of much lower redshift. The presence of such massive black holes in the early Universe implies that they must have grown very quickly. Observations show that high- $z$ blazars accrete matter at $\sim 10 \%$ of the Eddington rate (Ghisellini et al. 2010), indicating that they have already reached the end of the blazar formation process even at such a high redshift. (Ghisellini 2013).

Their broadband emission is dominated by relativistic jets and shows the typical double-hump structure of blazars (see Fig.1), but with the difference that the high-

michael@kreter.org energy peak rises above the synchrotron peak by one order of magnitude or more in power (Celotti \& Ghisellini 2008). Most of the known high- $z$ blazars have been detected in radio and optical surveys (Condon et al. 1998 Abolfathi et al. 2018). While blazars, in general, dominate the $\gamma$-ray sky (Abdollahi et al. 2020), the Large Area Telescope (LAT) onboard the Fermi satellite has only detected $\sim 12$ blazars at large $(z \geq 2.5)$ redshifts (Abdollahi et al. 2020, Acero et al. 2015). This discrepancy is caused by absorption on the extragalactic background light (EBL), which leads to an efficient degrading of high-energy $\gamma$ rays above $\sim 1 \mathrm{GeV}$ (Ackermann et al. 2017; H. E. S. S. Collaboration et al. 2013). This causes a suppression of the observed $\gamma$-ray emission from distant blazars. Along with absorption on the EBL, galaxy evolution could also explain the low number of high- $z$ blazars observed by Fermi/LAT (Ajello et al. 2014).

So far, the most distant known $\gamma$-ray emitting blazar is B3 $1428+422$ with a redshift of $z=4.72$ (Liao et al. 2018). Many of the other high- $z$ blazars observed by Fermi/LAT were only detectable when in bright flaring states (Paliya et al. 2019a). However, there is a lack of simultaneous broadband data, which is necessary for understanding their multiwavelength flaring behavior. Currently, the only high- $z$ blazars observed at multiple frequencies during a flare are TXS $0536+145(\mathrm{z}=2.69$; Orienti et al. 2014) and CGRaBS J0733 $+0456(z=3.01$; Liao et al. 2019). Their broadband emission is well described by a leptonic model, similar to models for blazars in the 


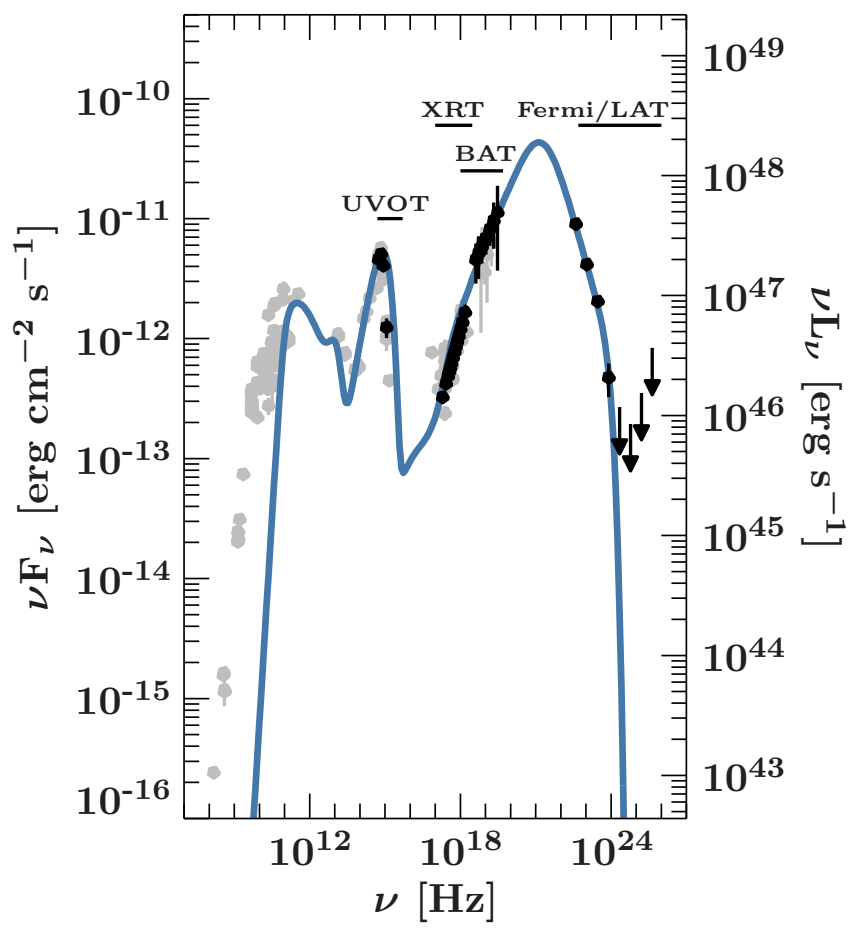

Figure 1. Example spectral energy distribution of the high- $z$ blazar DA $193(\mathrm{z}=2.365)$. These sources are typically weak at $\gamma$ ray energies, but show strong hard X-ray emission. Data adopted from Paliya et al. (2019a)

local Universe. Paliya et al. (2016, 2020); van den Berg et al. (2019); Marcotulli et al. (2020) studied spectral energy distributions (SEDs) of high- $z$ blazars, by using quasi-simultaneous datasets. All datasets could be well described by a simple one-zone leptonic model plus thermal emission from the accretion disk, with the emission from the high-energy hump originating from external Compton scattering.

Continuous monitoring of a chosen sample of sources in all available energy bands has revealed a correlation between the radio and $\gamma$-ray emission of blazars (e.g., Fuhrmann et al. 2014, Ackermann et al. 2011), which is expected to also be observed for high- $z$ blazars. Their high jet power and possibly different evolutionary state makes them particularly interesting targets, but so far no study on the correlation between radio and $\gamma$-ray emission is available for the most distant blazars. In the $\mathrm{keV}$ energy regime, the soft X-ray spectrum was observed to be flatter than expected for several high- $z$ sources (Eitan \& Behar 2013), which was first proposed to be due to intrinsic absorption of those sources (e.g., Fabian et al. 2001; Yuan et al. 2000). However, their optical/UV emission did not show this absorption, which created speculation about a so-called 'warm absorber', for which the absorbing material shows a huge gas-to-dust ratio and only affects the X-ray radiation (Fabian et al. 2001). Another possibility is an intrinsic curvature within the jet emission, which does not require a strong absorber to explain the observed soft X-ray deficit (e.g., Paliya et al. 2016, Foschini 2009, Tavecchio et al. 2007).

The number of known $\gamma$-ray emitting high- $z$ blazars was recently increased by Ackermann et al. (2017). These distant sources were detected by Fermi/LAT due to an extended energy range towards lower energies and

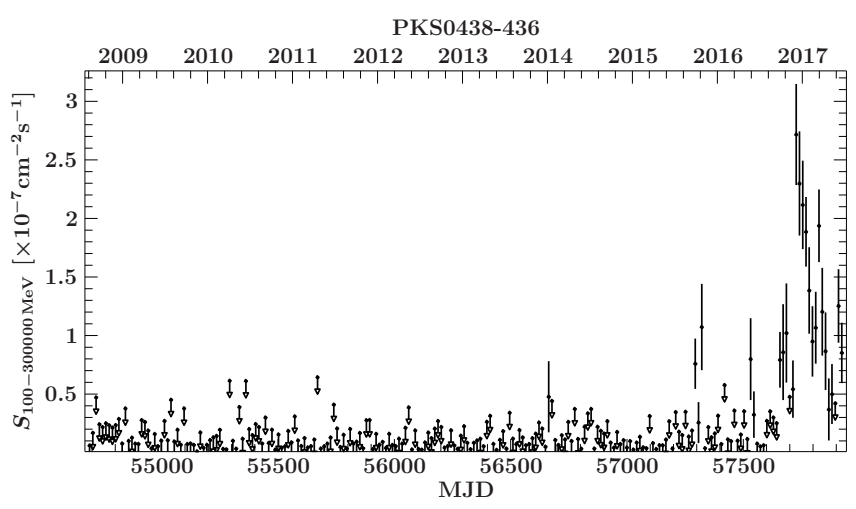

Figure 2. Fermi/LAT $\gamma$-ray light curve of PKS 0438-43 over about ten years of continuous observations. The light curve is shown for the energy range $100 \mathrm{MeV}$ to $300 \mathrm{GeV}$. This source was detected in December 2016 on daily time scales during a bright flare.

the employment of energy dispersion in the gamma-ray analysis, which allowed for the identification of $\mathrm{MeV}$ dominated blazars.

In this work, we introduce a new method to search for undetected high- $z$-ray emitting blazars that are too faint to be detected significantly on the long-term periods typically considered for catalogs, but can show significant emission during shorter $(\sim$ monthly $)$ periods. This paper is structured in the following way: In Sec.2.1, we introduce the selection criteria for the considered sample of radio and optically detected blazars. Sec.2.2 describes the details of the Fermi/LAT analysis performed. In Sec. 2.3 we discuss the approach employed to measure background fluctuations in the $\gamma$-ray sky and introduce statistical ways to calculate the probability of multiple low-significance ${ }^{1} \gamma$-ray detections from the same blazar. Sec. 3 focuses on $\gamma$-ray detections from the considered blazar sample at various test statistic (TS) levels The test statistic is defined as a likelihood ratio test according to $T S=-2 \log (L / L o)$. In Sec. 4 we compare our work to the first catalog of transient $\gamma$-ray sources observed by Fermi/LAT (1FLT) (Mereu et al. in prep) and discuss the limitations and statistical uncertainties of the method used. Furthermore, we present SEDs for the most significantly detected high- $z$ blazars and conclude in Sec. 5 .

\section{METHOD}

\subsection{Detection Strategy and Sample Selection}

Flaring activity from previously undetected high- $z \gamma$-ray blazars has been discovered several times by Fermi/LAT (Cheung 2016, Cheung 2017, Angioni 2018). Such periods of enhanced activity typically only last for several days, leading to a significant detection (TS $\geq 25$ ) due to a drastic background reduction compared to the long-term averaged fluxes used in catalogs. Figure 2 shows a ten-year Fermi/LAT, $\gamma$-ray light curve for the high-z blazar PKS 0438-43 $(\mathrm{z}=2.83)$ in the energy range $100 \mathrm{MeV}$ to $300 \mathrm{GeV}$. This source was not included in the third Fermi/LAT Source catalog and was detected at $\mathrm{GeV}$ energies only due to its bright flare in $2016^{2}$ (Cheung 2016).

\footnotetext{
1 TS $<25$ monthly intervals.

2 PKS 0438-43 is now included in the Fourth Fermi/LAT Source catalog (4FGL).
} 


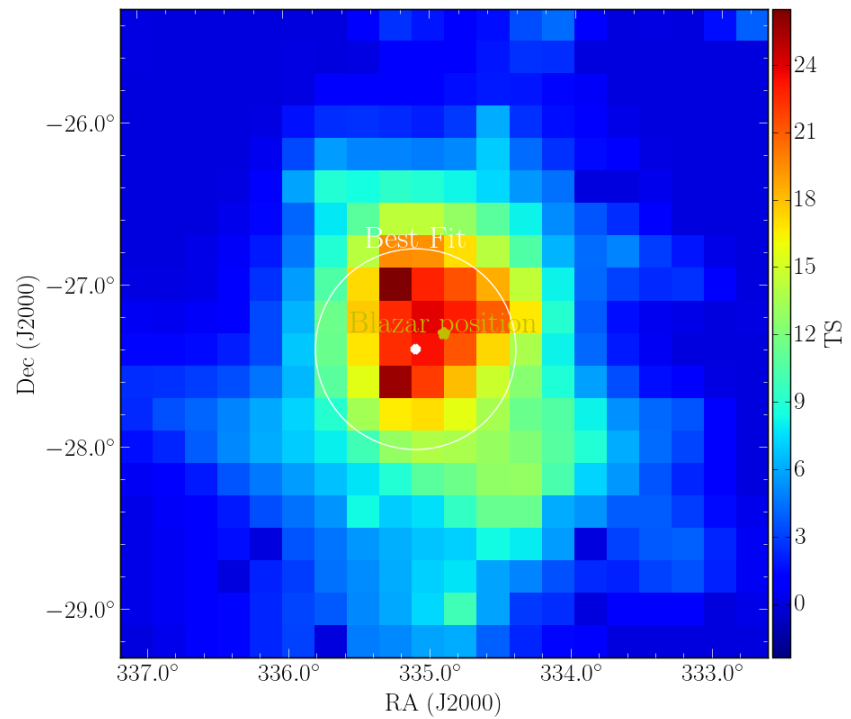

Figure 3. TS map of the blazar 5BZQ J2219-2719 at $\mathrm{z}=$ 3.63. The green star corresponds to the localization of the high- $z$ blazar using optical data. The white star and circle indicate the Fermi LAT best-fit position. An excess of $\sim 5 \sigma$ is seen, consistent with the target blazar.

In order to search for such flaring activity, we perform a systematic search for high- $z$ blazars that are not present in any Fermi/LAT catalog ${ }^{3}$, by targeting a sample of 176 radio and optically detected blazars with a redshift of $z \geq 2.5$ and a radio flux density of more than $50 \mathrm{mJy}$, taken from the Roma BZCAT Multifrequency Catalogue of Blazar ${ }^{4}$ and the SHAO list of high- $z$ radioloud quasar: 5 . Sources with a high radio flux density are selected due to a tight correlation between the radio and $\gamma$-ray flux (Ghirlanda et al. 2010, Ackermann et al. 2011). The total sample consists of 169 BZCAT blazars, and seven SHAO sources.

\subsection{Fermi/LAT data analysis}

For the analysis of the Fermi/LAT data we performed a dedicated likelihood and localization analysis of all blazars selected in Sec.2.1. Monthly binned Fermi/LAT $\gamma$-ray light curves in the time range from $2008 \mathrm{Au}$ gust 4 to 2019 April 4 and energy range of $100 \mathrm{MeV}$ to $300 \mathrm{GeV}$ were calculated, resulting in a total of 130 monthly intervals per blazar. We used the Fermi Science Tools (v11r5p3) together with the reprocessed Pass 8 data and the P8R3_SOURCE_V2 instrument response functions. We performed an unbinned likelihood analysis in a region of interest (ROI) of $10^{\circ}$ around the source of interest for each of these time bins. A zenith angle cut of $90^{\circ}$ was used together with the LAT EVENT CLASS $=128$ and the LAT EVENT TYPE $=3$, while the gtmktime cuts DATA_QUAL $=1 \& \&$ LAT $\mathrm{CONFIG}==1$ were chosen. For the modeling of the $\overline{\text { diffuse component }}{ }^{6}$ we used the Galactic diffuse

\footnotetext{
3 Since this project started before the release of the 4FGL catalog, we used the previous 3FGL Fermi/LAT source catalog. Some of the blazars that we found as part of our study were also found by the 4 FGL pipeline.

4 http://www.asdc.asi.it/bzcat/

5 http://202.127.29.4/CRATIV/en/high_z.html

6 https://fermi.gsfc.nasa.gov/ssc/data/access/lat/ BackgroundModels.html
}

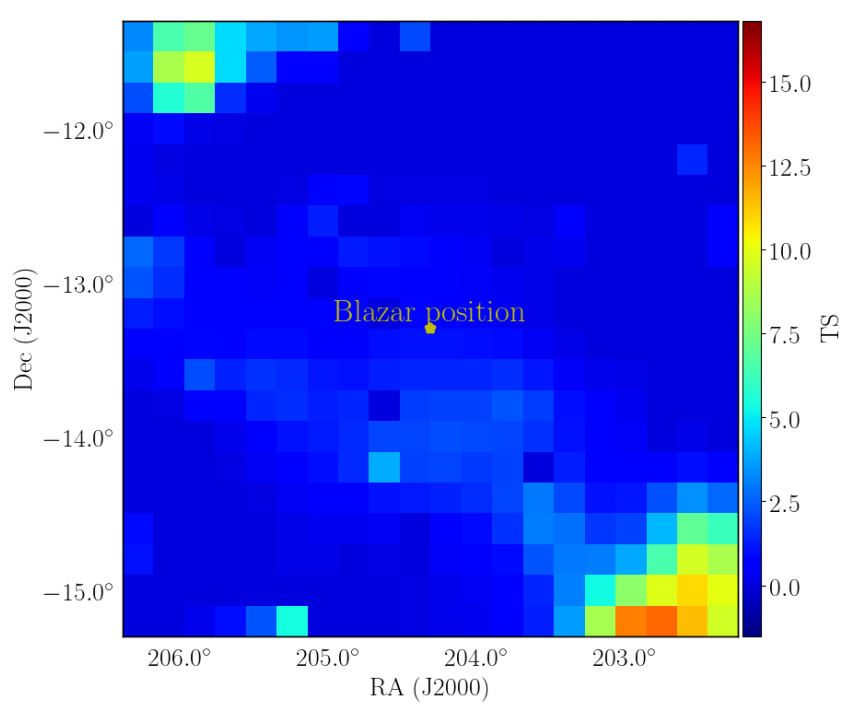

Figure 4. TS map of the blazar 5BZQ J1337-1319 at $\mathrm{z}=3.48$. The green star corresponds to the localization of the high- $z$ blazar using optical data. No excess at a position consistent with the target position is seen.

emission model gll_iem_v07.fits and the isotropic diffuse model iso P8R3 SOURCE V2 v01.txt. The model used in the likelihood analysis contained all of the sources from the Fourth Fermi/LAT Source catalog (4FGL; Abdollahi et al. 2020) within a radius of $20^{\circ}\left(\mathrm{ROI}+10^{\circ}\right)$ from the source of interest. In case the source of interest is not listed in the 4FGL catalog a new source is added, using the radio coordinates. If the studied high- $z$ blazar is already known to the 4FGL, this catalog source is used in the model. Sources were fitted with either a power-law or a log-parabola fit following the 4FGL catalog. New sources are always fitted with a power-law model. In the inner 10 degree from the source of interest, the parameters PREFACTOR and INDEX were left free to vary for each monthly interval, while for sources further outside all parameters were fixed to the 4 FGL values. The time binning of 30 days was chosen to study periods which would not have been monitored by the Fermi/LAT Flare Advocate service Ciprini \& Thompson 2013) while keeping the computing effort at a reasonable level. As a measurement of the detection significance of each monthly interval, we calculated the test statistic following Wilks' theorem (Wilks 1938; Mattox et al. 1996). In cases where no significant detection was found, upper limits were calculated based on the method of Feldman \& Cousins (1998).

Our localization analysis followed an iterative method using gtfindsrc. For each monthly time range we started with the radio coordinates to find the best-fit position. Those coordinates were then used as input for the next localization step. This iteration was repeated until the final best-fit position varied by less than $0.05^{\circ}$. In order to ensure that the observed emission could be associated to the previously undetected high- $z \gamma$-ray blazar, we searched for additional possible counterparts within the localization region. Since the majority of monthly intervals was at a low significance level of $\mathrm{TS}<9$, we considered only intervals for which the best-fit position stayed stable. In this way, a contamination of the observed $\gamma$-ray flux from other sources was prevented. TS 


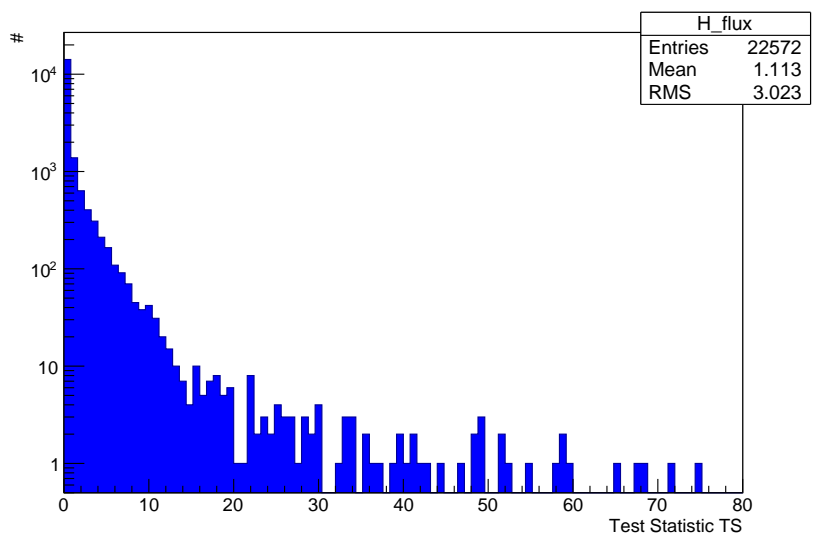

Figure 5. Test statistic distribution of 176 blazar positions selected according to Sec.2.1 22 monthly intervals are identified with a significance of TS $>25$, while at a TS $>9$ level 249 intervals are detected. The TS axis is scaled from zero to 80 .

maps were calculated for each monthly interval. Figure 3 shows such a map for the blazar 5BZQ J2219-2719 at Z $=3.63$. Monthly intervals for which no emission could be associated to the high- $z$ blazar best-fit position were not considered for further analysis. Fig. 4 shows such an excluded interval for the blazar 5BZQ $\sqrt{1337-1319}$ at $\mathrm{z}$ $=3.48$. Such excluded intervals are caused by a misidentification from a bright nearby $\gamma$-ray emitter.

\subsection{Background Determination}

At a test statistic level of TS $\geq 25 \gamma$-ray detections are considered as trustworthy, because the chance for a false positive detection is about $5 \times 10^{-7} \%$. Multiple $(\mathrm{TS}<25)$ detections from an unknown emitter, however, suggest the presence of a new $\gamma$-ray source, even if none of the monthly intervals are detected above the required $(\mathrm{TS}>25)$ threshold. In order to account for these sources, we measure the rate of false positive detections on monthly time scales over the entire $\gamma$-ray sky. This has not been done before. We perform a similar 7 Fermi/LAT light-curve analysis on a sample of blank sky positions. These positions have been randomly selected by mirroring the positions of known $\gamma$-ray blazars on the Galactic plane. In this way, the distribution of blank sky positions follows the observed blazar distribution in the sky. To make sure the measured false positive rate is not affected by nearby $\gamma$-ray sources, we exclude all random positions which are less then $1.5^{\circ}$ apart from any known Fermi/LAT source. Figure 6 shows the TS distribution of monthly intervals for the 80 blank-sky positions that were studied, while the TS distribution of the 176 high- $z$ blazars that were analyzed is shown in Fig.5. From the total number of blank-sky intervals studied, only a fraction of $11 / 9934 \approx 1.1 \times 10^{-3}$ monthly intervals showed a statistical fluctuation at a $\mathrm{TS} \geq 9$ level. This corresponds to a Gaussian standard deviation of about $3.26 \sigma$. In order to derive a representative measurement of random fluctuations over the entire $\gamma$-ray sky, it is necessary to perform this measurement for an extremely large number of blank sky positions. To account for the (always) finite number of positions analyzed, we used a Poisson fit of the TS distribution in Fig.6 to extrapolate the expected

7 The same energy, time range and binning are used.

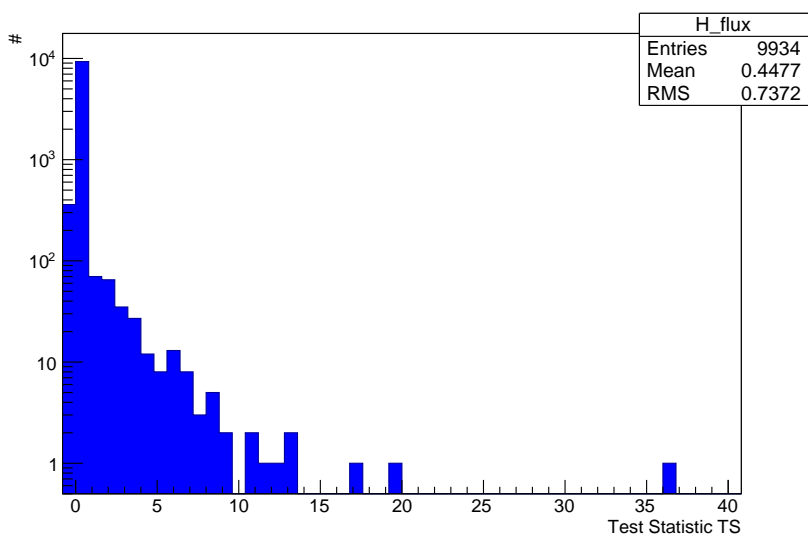

Figure 6. Test statistic distribution of a sample of 80 blank-sky positions. This histogram represents the background fluctuation for the performed Fermi/LAT variability analysis. The TS axis is scaled from zero to 40 .

background rate to higher TS values and to account for TS values without measured monthly intervals. In this way, the effect of large TS values in Fig.6, which might be caused by a miss identification of a bright source in the vicinity of the blank-sky position is reduced. By comparing the (normalized) number of measured fluctuations $\mu_{b}$ to the (normalized) observed number of monthly intervals $N_{s}$ detected from our sample of radio and optically selected high- $z$ blazars, we derive the Poisson probability $\Phi$ for a false positive detection of a detected monthly bin to be

$$
\Phi=P\left(N_{s} \mid \mu_{b}\right) .
$$

As a sanity check we solve Eqn. 1 for various test statistic levels. The results can be seen in Table1. We find a sample size of 80 blank-sky positions sufficient to keep this estimate stable. In order to determine whether the significance level of the signal measured from a blazar which shows multiple monthly bins with a TS $<25$ is sufficient to claim a detection, we consider the following two scenarios:

\subsubsection{Number of detections expected at $T S \geq 9$}

The probability that the entire observed $\gamma$-ray emission of individual blazars is purely below a detection threshold of $\mathrm{TS} \geq 9$ is

$$
P_{\geq N_{s}}=\sum_{n=N_{s}}^{N}\left(P_{9}\right)^{n} *\left(1-P_{9}\right)^{N-n}\left(\begin{array}{c}
N \\
n
\end{array}\right) .
$$

$P_{9}=1.1 \times 10^{-3}$ corresponds to the probability of a detection at a $3 \sigma$ level, while $N$ indicates the total number

Table 1

Poisson probability $\Phi$ of a single monthly light curve interval to be of purely statistical origin for various levels of test statistic.

\begin{tabular}{cc} 
TS value & $\Phi$ in $\sigma$ \\
\hline 10 & 3.26 \\
15 & 3.60 \\
25 & 4.88 \\
55 & 7.26 \\
\hline
\end{tabular}


Table 2

The five high-z blazars that were identified, each having been detected in a single monthly interval with TS $\geq 25$. Three of our identified blazars are known as new $\gamma$-ray sources due to the recently published 4FGL catalog, while two previously unknown $\gamma$-ray blazars have been identified. Four of the blazars found to have TS $\geq 25$ in at least one month were in the 3FGL and are therefore not listed in this table. The blazar 4FGL J0539.9-2839 has been included as a sanity check for the background estimate used.

\begin{tabular}{ccccccc}
$\begin{array}{c}\text { Source } \\
\text { Name }\end{array}$ & $\begin{array}{c}\text { RA } \\
\text { J2000 }\end{array}$ & $\begin{array}{c}\text { DEC } \\
\text { J2000 }\end{array}$ & $\mathbf{z}$ & $\begin{array}{c}\text { Detections } \\
\text { TS } \geq \mathbf{9}\end{array}$ & $\begin{array}{c}\text { Detections } \\
\text { TS } \geq \mathbf{2 5}\end{array}$ & $\begin{array}{c}\gamma \text {-ray } \\
\text { Counterpart }\end{array}$ \\
\hline 5BZQ J2219-2719 & 334.90 & -27.32 & 3.63 & 3 & 1 & $-/-$ \\
5BZQ J0422-3844 & 65.56 & -38.75 & 3.11 & 7 & 1 & $-/-$ \\
5BZQ J0733+0456 & 113.49 & 4.94 & 3.01 & 19 & 7 & 4FGL J0733.8+0455 \\
5BZQ J1127+5650 & 171.92 & 56.84 & 2.89 & 14 & 3 & 4FGL J1127.4+5648 \\
5BZQ J0434-4355 & 68.51 & -43.93 & 2.65 & 9 & 2 & 4FGL J0440.3-4333 \\
\hline 5BZQ J0539-2839 & 84.98 & -28.67 & 3.10 & 68 & 36 & 4FGL J0539.9-2839 \\
\hline
\end{tabular}

of monthly intervals per light curve and $N_{s}$ sets the number of $\mathrm{TS} \geq 9$ intervals. While the first two terms represent the number of light curve intervals observed with a $\mathrm{TS} \geq 9$ and vice versa, the last term $\left(\begin{array}{c}N \\ n\end{array}\right)$ takes the number of possible combinations with identical outcome into account. In this calculation, the exact TS value of each light curve interval is not considered. In the following, we choose the blazar BZQ J1430+4204 ( $\mathrm{z}=4.72)$ listed in Table 4 as an example of the statistical method used. This blazar has been identified in five monthly light curve intervals up to TS $\sim 20$. Following Eqn.2, this results in a Poisson probability of $P_{>N_{s}}=3.72 \sigma$. This probability does not correspond to the detection significance of this blazar, but rather to the probability of how likely it is that this source in general is detected at a TS $=9$ level. Table 3 lists the Poisson probability $P_{\geq N_{s}}$ for different numbers of TS $\geq 9$ intervals.

\subsubsection{Number of detections expected at each TS value}

To take the exact TS value of each light-curve interval into account, Eqn.2 is modified to

$$
P_{\text {tot }}=\sum_{i=1}^{N} \Phi_{i} *\left(1-\Phi_{i}\right)^{N-1} * 100
$$

Here, $\Phi_{i}$ corresponds to the Poisson probability calculated following Eqn. 1. The combinatoric factor is reduced to 100 , as each combination is unique and can occur at any of the bins of the blazar TS distribution. Applying Eqn.3 to BZQ J1430+4204 leads to a total probability of $P_{t o t}=6.57 \sigma$. In this calculation the exact TS value of each light curve interval has been considered.

By fixing $\Phi_{i}=P_{9}$ and allowing for multiple combinations, our result becomes equal to the probability derived in Sec.2.3.1. Nevertheless, $P_{t o t}$ represents the total probability of observing $\gamma$-ray emission from the studied

Table 3

Poisson probability $P_{\geq N_{s}}$ following Eq.2 for different numbers of $\stackrel{s}{T S} \geq 9$ intervals.

\begin{tabular}{cc}
$\mathbf{N}_{\mathbf{s}}$ & $P_{\geq N_{s}}$ in $\sigma$ \\
\hline 1 & 3.26 \\
2 & 3.38 \\
3 & 3.49 \\
4 & 3.61
\end{tabular}

blazar, considering the exact TS level of the measured $\gamma$-ray emission.

\subsection{Swift/XRT data analysis}

In our sample of significantly detected new $\gamma$-ray blazars listed in Table 4, 5BZQ J1429+5406 is the only source for which Swift/XRT data are available. These data were reduced with standard methods, using the software package HEASOFT v. 6.26.1. The data were reduced, calibrated and cleaned by means of the XRT-PIPELINE script using standard filtering criteria. We selected 0-12 grades for observations in photon counting (pc) mode. Spectral fitting was performed with ISIS 1.6.2 (Houck \& Denicola 2000). The data for 5BZQ J1429+5406 were extracted using XSELECT with a source radius of $54.218^{\prime \prime}$ centered on $\alpha=217.60, \delta=42.08$, in order to cover $\gtrsim 90 \%$ of the PSF at all energies. The background region was extracted from an annulus centered on the same coordinates, with radii of $82.506^{\prime \prime}$ and $235.731^{\prime \prime}$, while ensuring that no X-ray source was present in the background region. 5BZQ J1429+5406 shows a count rate of $0.04 \mathrm{cts} / \mathrm{s}$, which is not bright enough to raise any concerns about pile-up. Unfortunately, these data were not simultaneous with the observed $\gamma$-ray emission.

\section{INDIVIDUAL HIGH- $Z$ BLAZARS}

\section{1. $T S \geq 25$ detections}

Out of our sample of 176 high- $z$ blazars, five were detected in at least one monthly interval with a test statistic of $\mathrm{TS} \geq 25$. Table 2 lists the relevant properties of these blazars. Three blazars showed evidence for stronger emission in the first eight years of the Fermi mission and were, therefore, included in the 4FGL catalog. Two new high- $z$ blazars were identified as $\gamma$-ray blazars by the analysis performed here. They each showed evidence for heightened activity (TS $>25$ ) during one of the monthly time bins in the period after the eight-year time range considered for the 4FGL catalog. As can be seen in Table 2, the source 5BZQ J2219-2719, at a redshift of $\mathrm{z}=$ 3.63 , is identified as the most distant TS $>25$ blazar detected in our work. Besides their emission at a TS level of more then 25, these blazars showed multiple monthly intervals at a TS $\geq 9$ level. As a proof of principle of the performed background estimate, we included the known blazar PKS 0537-286 ( $\mathrm{z}=3.10)$ in our sample. This source has been detected multiple times at different significance levels on monthly time scales and even showed daily flaring activity (Cheung 2017). Following Eqn.3 for all monthly intervals with a $T S \geq 9$, we show that the 
Poisson probability that these detections are of purely random origin can be excluded at least with $P_{\text {tot }} \sim 8 \sigma$. This further proves that blazars at these large distances can be prominent $\gamma$-ray emitters.

\section{2. $T S \geq 9$ detections}

At a TS $\geq 9$ level, a total of 249 monthly intervals have been identified from 116 high- $z$ blazars. This suggests that the majority of blazars studied in this work show at least indications of $\gamma$-ray flaring activity. By comparing the individual TS distribution of the $116 \mathrm{TS} \geq 9$ blazars to the measured TS distribution of random fluctuations seen in Fig. 6 we find that 27 previously $\gamma$-ray undetected blazars show multiple monthly detections above the expected background. Table 4 lists the relevant properties of these blazars. While four of these blazars are in the 4FGL catalog due to increased levels of $\gamma$-ray emission after the time range considered in the 3FGL, 23 previously unknown high- $z$ blazar candidates have been identified, each with a total probability, $P_{\text {tot }}$, of at least $3 \sigma$. Out of these 23 potential new $\gamma$-ray blazars, six are detected with a $P_{\text {tot }}>5 \sigma$. In this sample of potential $\gamma$-ray blazars (TS $\geq 9$ in more than one monthly interval) at high redshift, 16 previously $\gamma$-ray undetected blazars are located at a redshift of $\mathrm{z} \geq 3$ with 6 of these blazars being even more distant at a redshift of $\mathrm{z} \geq 4$. The most distant blazars in our sample 5BZQ J0906 $+6930(\mathrm{z}=5.47)$ and 5BZQ J1026 $+2542(\mathrm{z}=5.29)$ had a signal at the TS 10 level during just one monthly interval each in the time period analyzed here. This is consistent with background fluctuations and therefore these sources are only considered as promising $\gamma$-ray candidates.

\section{DISCUSSION}

In this section, we compare our sample of new $\gamma$-ray blazars observed on monthly time scales to the first catalog of Fermi/LAT transients (1FLT), discuss limitations of the method used and present the $\gamma$-ray properties of these new high-energy emitters.

\subsection{Comparison to 1FLT catalog}

The 1FLT represents the first catalog of $\gamma$-ray transient sources detected on monthly time scales by Fermi/LAT. While general source catalogs like the 4FGL integrate over the entire mission time of the Fermi satellite (Abdollahi et al. 2020), the 1FLT searches for TS $>25$ detections on shorter time scales. Therefore, transient sources listed in the 1FLT are in general unknown to the longterm integrated catalogs.

Our results listed in Table 2 are fully consistent with the findings of the 1FLT catalog. In fact, only the new $\gamma$ ray detected blazar 5BZQ J2219-2719 is listed in the 1FLT, as this catalog does not include sources listed in the 4FGL and only covers a time range up to August 2018.

\subsection{Spectral properties}

Little is known about the radiative mechanisms of $\gamma$-ray loud blazars at high redshifts. In contrast to blazars in the local Universe, the SEDs of their distant relatives seem to be dominated by their high-energy peak in the blazar SED, which rises above the synchrotron peak by approximately one order of magnitude (Celotti
\& Ghisellini 2008). To understand the physical processes, which power this luminous subclass of distant AGN, multiwavelength studies of their spectral properties are crucial. Monthly $\gamma$-ray detections, as can be seen in Fig.2, have so far only been discovered months or even years after the detections, making simultaneous observations in other wavebands impossible. Building broadband SEDs for most high- $z$ blazars is therefore extremely challenging as the number of sources detected at different wavelengths is very limited. In fact, out of all new significantly detected $\gamma$-ray blazars ${ }^{8}$ listed in Table 2 and Table 4 , only a single blazar was observed at both X-ray and $\gamma$-ray energies. Figure 7 displays the high-energy hump of the blazar 5BZQ J1430+4204, using Swift/XRT and Fermi/LAT data.

The Fermi/LAT spectrum shows a soft power-law spectral index of 2.7, which is also common for blazars in the local universe during flaring states. The entire $\gamma$-ray emission is centered in the first spectral bin with $\mathrm{TS} \sim 12$. Such behavior is expected for $\gamma$-ray detections of high- $z$ blazars due to EBL scattering.

Swift/XRT data from 5BZQ J1430+4204 were fit using Cash statistics with a $\mathrm{S} / \mathrm{N}$ binning of 1 and an energy range of $0.3-10 \mathrm{keV}$. Fitting an absorbed power law yields a best-fit statistics of $\chi_{\text {reduced }}^{2}=0.88$ with 232 degrees of freedom. The best-fit power-law index is $\Gamma=1.18_{-0.10}^{+0.12}$, while the absorption has been fixed to the Galactic value of $9.34 \times 10^{19} \mathrm{~cm}^{-2}$. The unabsorbed $2-10 \mathrm{keV}$ flux is $1.73_{-0.23}^{+0.25} \times 10^{-12} \mathrm{erg} \mathrm{s}^{-1} \mathrm{~cm}^{-2}$. At $z=4.72$, this corresponds to a luminosity of $3.9 \pm 0.6 \times 10^{47} \mathrm{erg} \mathrm{s}^{-1}$.

\subsection{Limitations of the method used 4.3.1. Sample selection}

In this work, we presented a targeted search for $\gamma$-ray emission at high redshifts, based on a sample of 176 radio and optically selected blazars. Furthermore, only blazars with a certain radio flux density were selected ${ }^{9}$. This introduces a bias in the nature of detectable high- $z$ blazars and limits the potential of possible $\gamma$-ray detections. A more general search for $\gamma$-ray emitting high- $z$ blazars, including a trigger for quasi-simultaneous multiwavelength data will be the scope of our future work.

\subsubsection{Significance calculation}

In order to calculate the significance of a new blazar detection based on multiple TS $<25$ intervals, we systematically measured the amount of random fluctuations in the entire $\gamma$-ray sky on monthly time scales. To account for the (always) finite number of blank-sky positions studied, we normalize the TS distributions of our high- $z$ blazar sample seen in Fig. 5 as well as the TS distribution of the blank-sky positions studied, as seen in Fig.6. Furthermore, we fit these distributions with a Poisson function 10 to account for TS values that have not been measured. To calculate the uncertainty of the total detection probability $\Delta P_{t o t}$, we vary the parameters of the Poisson fit. We also tested different functions (Gaussian and exponential) to quantify how well they described the measured TS distributions. We find that the total detection

\footnotetext{
8 This includes the two new sources in Table 2 as well as the six blazars in Table 4 which show a $P_{t o t}>5 \sigma$.

${ }^{9}$ Please refer to Sec. 2.1 for further details.

10 Please refer to Sec.2.3 for further details.
} 
probability $P_{t o t}$ differs by about $2 \%$ to $10 \%$ for different fit functions or parameters. Thus, we estimate the systematic uncertainty of the presented significance calculation to be on the order of $\sim 10 \%$.

\subsubsection{Counterpart association}

Within the localization uncertainty of the high- $z$ blazars studied (see e.g. Fig. 3), additional $\gamma$-ray counterparts could also be present. Since we only consider multiple light-curve intervals for those objects whose best-fit positions stay stable at the same coordinates, a contamination from unrelated $\gamma$-ray emitters within the localization uncertainty seems to be unlikely. However, even if the positional variance is low, a misidentification of other $\gamma$ ray emitting sources consistent with the best-fit position can not be fully ruled out. Therefore, we consider the identified high- $z$ blazars as the most plausible counterparts to the observed $\gamma$-ray emission.

\section{CONCLUSION}

We have studied the long-term $\mathrm{MeV}-\mathrm{GeV}$ variability behavior of a sample of 176 radio and optically detected high- $z$ blazars using Fermi/LAT. A dedicated localization analysis has been performed to ensure an association between the observed $\gamma$-ray emission and the targeted high- $z$ blazars. Indications of $\gamma$-ray emission have been detected from more than half of the blazar sample studied. The two previously unknown $\gamma$-ray blazars 5BZQ J2219-2719 ( $\mathrm{z}=3.63)$ and 5BZQ J0422-3844 (z $=3.11$ ) have each been identified in a single monthly interval at a significance level of $\mathrm{TS} \geq 25$. In addition, we have confirmed, at the TS $>25$ level, $\gamma$-ray emission from three 4FGL blazars on monthly timescales. These only showed enhanced $\gamma$-ray activity in recent years and were, therefore, not listed in the 3FGL catalog. In order to account for the huge detection potential of multiple $\mathrm{TS}<25$ light curve intervals, we have systematically studied the expected number of all-sky random fluctuations on monthly time scales. To calculate the total detection probability $P_{t o t}$ (see Eqn.3) of multiple TS $<25$ detections, we compared the number of measured light curve intervals of the blazars considered to the number of expected monthly fluctuations at each TS value. Table 4 lists the results for 27 blazars which have been identified in multiple low-significance detections. This includes 23 blazars previously undetected at $\gamma$-ray energies. Four of these blazars were not in the 3FGL catalog but were included in 4FGL, the most recent Fermi/LAT source catalog. This demonstrates our method to be robust, as multiple low-significance detections provide a clear indication for a new $\gamma$-ray source.

Six blazars listed in Table 4 have been identified with a $P_{t o t} \geq 5 \sigma$, and are marked in bold. These include the most distant blazars studied in our sample. The two blazars 5BZQ J1430+4204 $(\mathrm{z}=4.72)$ and 5BZU J0525-3343 $(\mathrm{z}=4.41)$ lie at redshifts more distant than previously reported $\gamma$-ray blazars, making them the most distant blazars ever detected by Fermi/LAT. In contrast to the blazars reported by Ackermann et al. (2017), which were detected in an integrated analysis over 92 months of LAT data, the long-term integrated flux of our sources is too faint for a significant source detection over long time scales.

Our results are consistent with the findings of Liao et al.

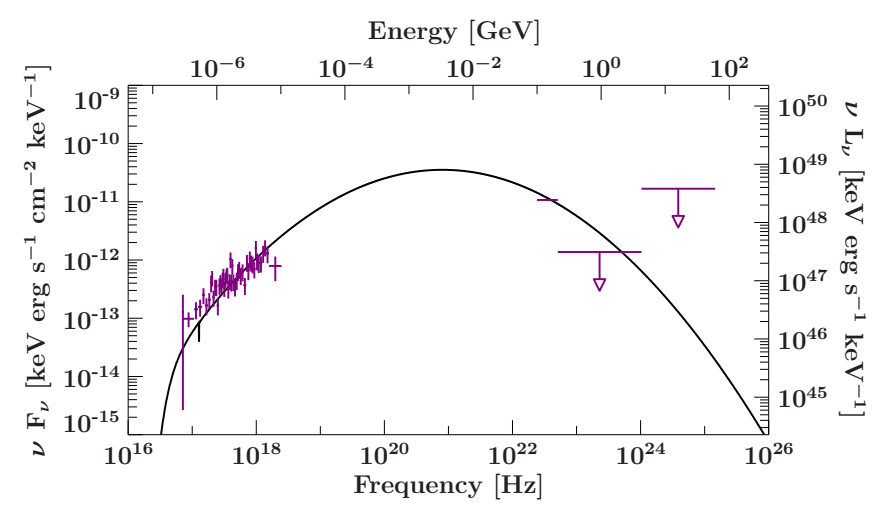

Figure 7. Multiwavelength SED of 5BZQ J1430 $+4204(\mathrm{z}=4.72)$, using Swift/XRT and Fermi/LAT data. The blazar high-energy hump is modeled with a log parabola. The entire emission observed by Fermi/LAT is centered on the $\sim 100 \mathrm{MeV}$ bin with a test statistic of $\mathrm{TS} \sim 12$.

(2018), who independently confirmed $\gamma$-ray emission observed by Fermi/LAT from the direction of the blazar 5BZQ J1430+4204. While Liao et al. (2018) claim a flaring period of about 10 months in the time range of July 2012 to May 2013, our variability analysis indicates that it was only during three of the monthly intervals in this time period that 5BZQ J1430+4204 showed evidence for $\gamma$-ray emission at TS $\sim 12$. Furthermore, we also find indications of enhanced $\gamma$-ray activity in monthly bins at a TS $\sim 15$ level covering a time range from December 2014 to January 2015.

This differences in both analyses are likely due to the use of different sky models and instrument response functions. While Liao et al. (2018) use the preliminary Fermi/LAT 8 year Source List (FL8Y ${ }^{11}$ ), our work is based on the most recent general source cata$\log$ (4FGL). In an independent analysis, Li et al. (2020) confirmed transient $\gamma$-ray activity from the two blazars 5BZQ J2219-2719 and 5BZQ J2321-0827, respectively. These sources account for two of the most significant detected new blazars in our sample. By studying flaring periods of several months, Li et al. (2020) was able to identify both blazars with a TS $>25$.

In addition to the previously discussed high- $z$ blazar identifications, indications of high-energy emission at a $P_{\text {tot }}>4 \sigma$ level have been identified from 36 blazars, including 16 blazars previously undetected by Fermi/LAT. Two blazars, 5BZQ J0906+6930 ( $\mathrm{z}=5.47)$ and 5BZQ J1026 $+2542(\mathrm{z}=5.29)$, each showed evidence for emission during a single monthly time interval with a TS of $\sim 10$. Since our analysis has shown that single detections at this low-significance level are fully consistent with random fluctuations, we do not consider these two blazars as $\gamma$-ray emitters.

In Sec.4.2, we studied the spectral properties of our most distant significantly detected blazar 5BZQ J1430 +4204 $(\mathrm{z}=4.72)$. Figure 7 displays the high-energy peak of the blazar SED, using Swift/XRT and Fermi/LAT data. The X-ray through $\gamma$-ray SED is very poorly constrained, but does not show obvious signs of unusual high-energy spectral components different from the high-energy SEDs in lower-redshift blazars (Fan et al. 2016).

Due to the sparse multiwavelength coverage of most of the blazars studied, (quasi)-simultaneous SEDs could

\footnotetext{
11 https://fermi.gsfc.nasa.gov/ssc/data/access/lat/fl8y/
} 
not be constructed. Due the the large distance of these sources, however, the $\gamma$-ray emission peaks towards longer wavelengths, causing a more pronounced increase in the emission in the $\mathrm{keV}-\mathrm{MeV}$ regime. Future missions like the All-sky Medium Energy Gamma-ray Observatory (AMEGO) will provide a substantial contribution to the study of blazars in the early Universe (Paliya et al. 2019b). Quasi-simultaneous multiwavelength data of high- $z$ blazars would provide crucial insights into the spectral properties and emission behaviors of blazars which existed so early in the Universe. A detailed study of (quasi)-simultaneous MWL observations of flaring high- $z$ blazars will be the focus of our future work.

The work of M. Kreter and M. Böttcher is supported by the South African Research Chairs Initiative (grant no. 64789) of the Department of Science and Innovation and the National Research Foundation ${ }^{12}$ of South Africa. A. Gokus was partially funded by the Bundesministerium für Wirtschaft und Technologie under Deutsches Zentrum für Luft- und Raumfahrt (grant 50OR1607O) and by the German Science Foundation (DFG, grant KR 3338/4-1). F. Krauß was supported as an Eberly Research Fellow by the Eberly College of Science at the Pennsylvania State University. We thank J.E. Davis for the development of the slxfig module that has been used to prepare the figures in this work. This research has made use of a collection of ISIS scripts provided by the Dr. Karl Remeis-Observatory, Bamberg, Germany at http://www.sternwarte.uni-erlangen. de/isis/. The Fermi LAT Collaboration acknowledges generous ongoing support from a number of agencies and institutes that have supported both the development and the operation of the LAT as well as scientific data analysis. These include the National Aeronautics and Space Administration and the Department of Energy in the United States, the Commissariat à l'Energie Atomique and the Centre National de la Recherche Scientifique / Institut National de Physique Nucléaire et de Physique des Particules in France, the Agenzia Spaziale Italiana and the Istituto Nazionale di Fisica Nucleare in Italy, the Ministry of Education, Culture, Sports, Science and Technology (MEXT), High Energy Accelerator Research Organization (KEK) and Japan Aerospace Exploration Agency (JAXA) in Japan, and the K. A. Wallenberg Foundation, the Swedish Research Council and the Swedish National Space Board in Sweden. Additional support for science analysis during the operations phase is gratefully acknowledged from the Istituto Nazionale di Astrofisica in Italy and the Centre National d'Études Spatiales in France. This work performed in part under DOE Contract DE-AC02-76SF00515. We acknowledge the use of public data from the Swift data archive. We thank Vaidehi Paliya for providing the broadband SED data of DA 193 shown in Fig.1.

\section{REFERENCES}

\footnotetext{
12 Any opinion, finding and conclusion or recommendation expressed in this material is that of the authors and the NRF does
}

Abdollahi, S., Acero, F., Ackermann, M., et al. 2020, ApJS, 247, 33

Abolfathi, B., Aguado, D. S., Aguilar, G., et al. 2018, ApJS, 235, 42

Acero, F., Ackermann, M., Ajello, M., et al. 2015, ApJS, 218, 23

Ackermann, M., Ajello, M., Allafort, A., et al. 2011, ApJ, 741, 30

Ackermann, M., Ajello, M., Allafort, A., et al. 2011, ApJ, 741, 30

Ackermann, M., Ajello, M., Baldini, L., et al. 2017, ApJ, 837, L5

Ajello, M., Costamante, L., Sambruna, R. M., et al. 2009, ApJ, 699,603

Ajello, M., Romani, R. W., Gasparrini, D., et al. 2014, ApJ, 780, 73

Angioni, R. 2018, The Astronomer's Telegram, 11137

Bloemen, H., Bennett, K., Blom, J. J., et al. 1995, A\&A, 293, L1

Celotti, A., \& Ghisellini, G. 2008, MNRAS, 385, 283

Cheung, C. 2016, The Astronomer's Telegram, 9854

Cheung, C. 2017, The Astronomer's Telegram, 10356

Ciprini, S., \& Thompson, D. J. 2013, arXiv e-prints, arXiv:1303.4054

Condon, J. J., Cotton, W. D., Greisen, E. W., et al. 1998, AJ, 115,1693

Eitan, A., \& Behar, E. 2013, The Astrophysical Journal, 774, 29

Fabian, A. C., Celotti, A., Iwasawa, K., \& Ghisellini, G. 2001,

Monthly Notices of the Royal Astronomical Society, 324, 628

Fabian, A. C., Celotti, A., Iwasawa, K., et al. 2001, MNRAS, 323, 373

Fan, J. H., Yang, J. H., Liu, Y., et al. 2016, ApJS, 226, 20

Feldman, G. J., \& Cousins, R. D. 1998, Phys. Rev. D, 57, 3873

Foschini, L. 2009, Advances in Space Research, 43, 1036

Fuhrmann, L., Larsson, S., Chiang, J., et al. 2014, MNRAS, 441, 1899

Ghirlanda, G., Ghisellini, G., Tavecchio, F., \& Foschini, L. 2010, MNRAS, 407, 791

Ghisellini, G. 2013, Mem. Soc. Astron. Italiana, 84, 719

Ghisellini, G., Della Ceca, R., Volonteri, M., et al. 2010, MNRAS, 405, 387

H. E. S. S. Collaboration, Abramowski, A., Acero, F., et al. 2013, A\&A, 550, A4

Houck, J. C., \& Denicola, L. A. 2000, ISIS: An Interactive Spectral Interpretation System for High Resolution X-Ray Spectroscopy

Li, S., Sun, L.-M., Liao, N.-H., \& Fan, Y.-Z. 2020, arXiv e-prints, arXiv:2006.10470

Liao, N.-H., Dou, L.-M., Jiang, N., et al. 2019, ApJ, 879, L9

Liao, N.-H., Li, S., \& Fan, Y.-Z. 2018, ApJ, 865, L17

Marcotulli, L., Paliya, V., Ajello, M., et al. 2020, arXiv e-prints, arXiv:2001.01956

Mattox, J. R., Bertsch, D. L., Chiang, J., et al. 1996, ApJ, 461, 396

Mereu, I., Cavazzuti, E., Cutini, S., \& Tosti, G. in prep, A\&A

Orienti, M., D'Ammando, F., Giroletti, M., et al. 2014, MNRAS, 444, 3040

Paliya, V. S., Ajello, M., Cao, H. M., et al. 2020, ApJ, 897, 177

Paliya, V. S., Parker, M. L., Fabian, A. C., \& Stalin, C. S. 2016, ApJ, 825, 74

Paliya, V. S., Ajello, M., Ojha, R., et al. 2019a, ApJ, 871, 211

Paliya, V. S., Ajello, M., Marcotulli, L., et al. 2019b, arXiv e-prints, arXiv:1903.06106

Tavecchio, F., Maraschi, L., Ghisellini, G., et al. 2007, The Astrophysical Journal, 665, 980

Toda, K., Fukazawa, Y., \& Inoue, Y. 2020, arXiv e-prints, arXiv:2005.02648

van den Berg, J. P., Böttcher, M., Domínguez, A., \& López-Moya, M. 2019, ApJ, 874, 47

Wilks, S. S. 1938, Annals Math. Statist., 9, 60

Yuan, W., Matsuoka, M., Wang, T., et al. 2000, ApJ, 545, 625 
SEARCH FOR HIGH-REDSHTáblBuAZARS WITH Fermi/LAT

The 27 high- $z$ blazars that were identified, each having been detected with TS $>9$ in more than one monthly interval. The sources listed in Table 2 as well as mis-identified sources and blazars that were listed in 3FGL have been excluded. Out of this sample, 23 sources do not show any known $\gamma$-ray counterpart. Significantly identified previously undetected high- $z$ blazars are marked in bold.

\begin{tabular}{|c|c|c|c|c|c|c|}
\hline $\begin{array}{l}\text { Source } \\
\text { Name }\end{array}$ & $\begin{array}{c}\text { RA } \\
\text { J2000 }\end{array}$ & $\begin{array}{c}\text { DEC } \\
\text { J2000 }\end{array}$ & $\mathbf{z}$ & $\begin{array}{c}\text { Detections } \\
\text { TS } \geq 9\end{array}$ & $\begin{array}{c}\mathbf{P}_{\text {tot }} \\
{[\sigma]}\end{array}$ & $\begin{array}{c}\gamma \text {-ray } \\
\text { Counterpart }\end{array}$ \\
\hline 5BZQ J1429+5406 & 217.34 & 54.10 & 3.01 & 6 & 7.75 & 4FGL J1428.9+5406 \\
\hline 5BZQ J2321-0827 & 350.33 & -8.46 & 3.16 & 5 & 6.84 & $-/-$ \\
\hline $5 B Z Q \mathrm{~J} 1430+4204$ & 217.60 & 42.08 & 4.72 & 5 & 6.57 & $-/-$ \\
\hline 5BZU J0525-3343 & 81.28 & -33.72 & 4.41 & 4 & 5.73 & $-/-$ \\
\hline 5BZQ J1124-1501 & 171.01 & -15.03 & 2.56 & 3 & 5.50 & 4FGL J1122.5-1440 \\
\hline $5 B Z Q$ J0741+2557 & 115.37 & 25.96 & 2.75 & 3 & 5.49 & $-/-$ \\
\hline 5BZQ J1424+2256 & 216.16 & 22.93 & 3.62 & 4 & 5.25 & $-/-$ \\
\hline 5BZQ J1007+1356 & 151.92 & 13.94 & 2.72 & 3 & 5.24 & $-/-$ \\
\hline 5BZQ J0220-2151 & 35.15 & -21.85 & 2.55 & 2 & 4.53 & $-/-$ \\
\hline 5BZQ J0530-2503 & 82.53 & -25.06 & 2.78 & 2 & 4.52 & 4FGL J0529.1-2449 \\
\hline 5BZQ J0839+5112 & 129.94 & 51.20 & 4.40 & 2 & 4.52 & $-/-$ \\
\hline 5BZQ J1949-1957 & 297.47 & -19.95 & 2.65 & 2 & 4.52 & $-/-$ \\
\hline 5BZQ J1740+4506 & 265.03 & 45.11 & 2.79 & 2 & 4.51 & $-/-$ \\
\hline 5BZQ J1942-7015 & 295.69 & -70.26 & 2.90 & 2 & 4.50 & $-/-$ \\
\hline 5BZQ J0324-2918 & 51.18 & -29.31 & 4.63 & 2 & 4.49 & $-/-$ \\
\hline 5BZU J2248-0541 & 342.00 & -5.69 & 3.30 & 2 & 4.48 & $-/-$ \\
\hline 5BZQ J1616+0459 & 244.16 & 4.99 & 3.22 & 2 & 4.48 & $-/-$ \\
\hline 5BZQ J0121-2806 & 20.25 & -28.11 & 3.12 & 2 & 4.48 & $-/-$ \\
\hline 5BZQ J1413+4505 & 213.33 & 45.09 & 3.11 & 2 & 4.48 & $-/-$ \\
\hline 5BZQ J1421-0643 & 215.28 & -6.73 & 3.69 & 2 & 4.48 & $-/-$ \\
\hline 5BZQ J1100-4249 & 165.21 & -42.82 & 3.56 & 3 & 4.47 & $-/-$ \\
\hline SHAO J0257+4338 & 44.50 & 43.64 & 4.07 & 2 & 4.47 & $-/-$ \\
\hline 5BZQ J2015+6554 & 303.98 & 65.91 & 2.85 & 2 & 4.47 & 4FGL J2015.4+6556 \\
\hline 5BZQ J0001+1914 & 0.29 & 19.24 & 3.10 & 2 & 4.46 & $-/-$ \\
\hline 5BZQ J0612-4337 & 93.12 & -43.63 & 3.46 & 3 & 4.46 & $-/-$ \\
\hline 5BZQ J0918+0636 & 139.60 & 6.61 & 4.22 & 2 & 4.45 & $-/-$ \\
\hline 5BZQ J1139-1552 & 174.87 & -15.88 & 2.63 & 2 & 3.53 & $-/-$ \\
\hline
\end{tabular}

